УДК 621.311.25: 621.311.26

\title{
МОДЕЛИРОВАНИЕ ЗАКОНОВ РАСПРЕДЕЛЕНИЙ МОЩНОСТИ ВЕТРОЭНЕРГЕТИЧЕСКИХ И СОЛНЕЧНЫХ СТАНЦИЙ
}

\author{
Манусов Вадим Зиновьевич1, \\ Manusov36@mail.ru \\ Лемешко Борис Юрьевич 1 , \\ lemeshko@ami.nstu.ru \\ Халдаров Шерзод Камалханович 1 , \\ haldaroff@mail.ru \\ 1 Новосибирский государственный технический университет, \\ Россия, 630073, г. Новосибирск, пр. Карла Маркса, 20.
}

\begin{abstract}
Актуальность. Мощность, выдаваемая генерирующими станциями на возобновляемых источниках энергии, ветроэнергетическими и солнечными, зависит от состояния природной среды в конкретной географической точке, доминирующих скоростей ветра и уровня солнечной инсоляции. Закономерности, характеризующие скорости ветра и солнечную инсоляцию, зависят от местности, времени года и носят вероятностньй характер. Это значит, что вероятностный характер имеет и мощность, выдаваемая генерирующими станциями на возобновляемых источниках энергии. Риски, связанные с неточным знанием вероятностных закономерностей, описывающих эти источники, а также непредсказуемость (неопределённость) природной среды являются причинами, из-за которых системные операторы не учитьвают такие станции в расчетах баланса мощности. Это, в свою очередь, не позволяет рассматривать генерирующие станции на возобновляемой энергии как полноценных участников рынка, прикрывая поток инвестиций в подобные проекты. Уточнение законов распределения вероятнностей скорости ветра и солнечной инсоляции на основании статистического анализа соответствующих рядов измерений, а также уточнение на их основе законов распределения вероятностей для мощности соответствующих электростанций может оказать существенную помощь системному оператору при прогнозировании мощности, генерируемой станциями на «зеленой» энергии.

Цель: построение моделей законов распределения, наилучшим образом описывающих результаты наблюдений скорости ветра и солнечной инсоляции, а также моделей законов распределения для вырабатываемой мощности ветроэнергетической и солнечной станций.

Методы: методы оценки параметров законов распределения, методы проверки статистических гипотез.

Результаты. Показано, что в отличие от наиболее часто используемого в этих иелях закона Вейбулла, наилучшими моделями законов распределения вероятностей для рядов измерения скорости ветра и солнечной инсоляции на рассматриваемой территории являются законы семейства бета-распределений 3-го рода, а также смеси этих законов. То же самое касается описания распределения вероятностей мощности ветроэнергетической и солнечной станций. Построены модели законов распределения (по месяцам) для скорости ветра, солнечной инсоляции и мощности соответствующих станций. Показано, что на основании закона распределения скорости ветра и математических соотношений, связывающих мощность со скоростью ветра, можно строить хорошие модели для распределений вероятностей мощности ветроэнергетических станций. Построенные модели могут использоваться в качестве вспомогательного инструмента для системньх операторов энергосистем для прогнозирования выдаваемой мощности.
\end{abstract}

\section{Ключевые слова:}

Законы распределения вероятностей, возобновляемые источники энергии, распределение вероятностей выдаваемой мощности, бета-распределения III рода.

\section{Введение}

Несмотря на существенные плюсы, связанные с использованием возобновляемых источников энергии (ВИЭ) на ветровых и солнечных электрических станциях, одним из основных минусов остается плохая прогнозируемость выработки электроэнергии такими станциями, что связано со стохастическим характером ВИЭ. Этот факт доставляет массу неудобств системному оператору энергосистемы, как основному регулятору на рынке электроэнергии и мощности. Он сдерживает также возможные инвестиции в «зеленые проекты». При проектировании приходится учитывать, что выработка электроэнергии на солнечной и ветровой электростанциях, зависящих от солнечной радиации и скорости ветрового потока, не совпадает по времени, и в совокупности их выработка может не совпадать по времени с распределением спроса на электроэнергию. Очевидно, что проектирование и использование таких систем независимо друг от друга делает каждый из проектов более дорогостоящим из-за бо̀льших затрат на накопители энергии [1], а также приводит к снижению надежности общей системы. Более предпочтительным представляется использование гибридных систем в сочетании с накопителями энергии уже с меньшими габаритами. Это может сделать проекты энергосистем на ВИЭ более привлекательными для инвесторов.

Однако в гибридной системе генерируемая мощность также носит стохастический характер, определяемый вероятностными закономерностями, связанными с ВИЭ. Поэтому и здесь возможности прогнозирования, как правило, ограничиваются отсутствием 
информации о вероятностных закономерностях, описывающих генерируемую мощность, или качеством этой информации.

\section{Постановка задачи}

Вопросам оптимального проектирования гибридных энергетических систем на возобновляемых источниках энергии в целях повышения их доли в выработке электроэнергии и снижения неопределённости в величине генерируемой мощности к настоящему времени посвящено достаточно много работ Например, в [2] сделана попытка спрогнозировать выработку мощности ветроэнергетической станции с помощью детерминированных и вероятностных методов с целью сделать режим работы таких станций более предсказуемым и приближенным к традиционным генерациям. В работе [3] рассмотрена разработка алгоритма, рассчитывающего оптимальные параметры и габариты солнечной электростанции. В работах $[4,5]$ рассматриваются определение габаритов и затрат на строительство автономной гибридной электростанции на солнечной и ветровой энергии с помощью численных методов. В [6] для проектирования оптимальной гибридной энергосистемы на ВИЭ, работающей как автономно, так и параллельно с энергосистемой, используется линейное программирование. В данной работе авторы сделали попытку оптимизировать систему с точки зрения габаритов и затрат на строительство станции. В $[7,8]$ методами статистического анализа подбираются функции распределения вероятностей для вырабатываемой мощности на солнечной и ветровой электростанциях и делается попытка спроектировать подобную гибридную энергосистему. В [9] рассматриваются функции распределения для индекса ясности и возможные приложения для них.

Во многих работах, как, например, в [10], для описания скорости ветра наиболее подходящим считают закон распределения Вейбулла.

В [11] для вероятностного описания скоростей ветра используется распределение Вейбулла, а для описания солнечной инсоляции - гаммараспределения.

По мнению авторов работы [12], для математического описания ветрового режима юго-востока европейской части территории России целесообразно использовать распределение Вейбулла-Гудвича, что при расчете ветроэнергетических ресурсов территории позволит достичь положительного эффекта.

В [13] при анализе результатов измерений скорости ветра, выполненных за четырехлетний период на четырех географических станциях в Бурунди в процессе построения моделей законов распределения предпочтение было отдано бета-распределению 1-го рода.

В [14] при выборе для описания скоростей ветра законов распределения Вейбулла, Рэлея, логнормального, нормального и гамма-распределения адекватность построенных моделей анализировалась графически и проверялась с использованием критерия $\chi^{2}$.
В последнее время в исследованиях энергетического потенциала скорости ветра и солнечной радиации при описании вероятностных свойств источников стали использовать более сложные вероятностные модели и больше внимания уделяют проверке адекватности моделей. При этом заметно возрастает число публикаций в этой области.

В работе [15] множество рассматриваемых законов расширилось применяемыми обобщенным распределением экстремальных значений и распределением Накагами, в [16] - расширенным обобщённым распределением Линдли. В [17] дополнительно рассматриваются обратное гауссовское распределение и обобщенное логистическое, а также говорится о построении моделей в виде смесей известных параметрических законов. В работе [18] при построении моделей рассматривается уже 21 параметрический закон и предусматривается построение моделей в виде смесей этих законов. Здесь же рассматриваются вопросы применения различных критериев согласия для проверки адекватности моделей. В [19] подчеркивается, что от точности построения моделей законов для скорости ветра зависит точность прогнозирования средней мощности, выдаваемой ветряной турбиной. Здесь же в качестве возможных моделей дополнительно рассматриваются обобщенное распределение экстремальных значений, каппа-распределение и распределение Уэйкби.

В работе [20] делается попытка решения уже более сложной задачи, связанной с моделированием трехмерного ветрового ресурса Германии, в которой рассматривается модель скорости ветра - сдвига ветра. В модели опираются на данные о скорости ветра у поверхности и данные о скорости ветра при повторном анализе.

В [21] рассматриваются проблемы, связанные с применением различных критериев для проверки адекватности моделей для скорости ветра.

Исследование распределения вероятностей почасового солнечного облучения для города Кумаси в Гане в [22] проводилось с использованием данных за 14 лет. Анализ проводился для выявления закона распределения, наилучшим образом описывающего измерения для каждого месяца в году. В качестве наилучших моделей использовались законы: экспоненциальный, Вейбулла, логнормальный, гаммараспределения и геометрическое распределение. В работе [23] в качестве вероятностных моделей для инсоляции используются законы распределения Вейбулла, нормальное, логнормальное и гаммараспределение, в [24] упор делается на применение бета-распределения 1-го рода, а в [25] дополнительно рассматривается использование распределений логистического, экстремальных значений, обобщенного распределения экстремальных значений. Авторы работы [25] в результате статистического анализа обширных данных солнечной инсоляции за 2000-2008 гг. в районе города Ибадан в Нигерии пришли к заключению, что из множества рассмотренных ими законов наиболее подходящей моделью оказывается логистический закон распределения вероятностей. 
Анализ проводимых исследований и опубликованных результатов позволяет говорить о наличии проблем как при построении вероятностных моделей для скорости и солнечной инсоляции, так и при проверке адекватности этих моделей с использованием статистических критериев. Коротко их можно охарактеризовать следующим образом. Во-первых, построить хорошую модель для описания, например, скорости ветра в определённом месте в рамках некоторых простых параметрических моделей можно лишь в редких случаях. Необходимо использовать более широкий круг параметрических законов с возможностью построения моделей в виде смесей этих законов. Вовторых, при проверке адекватности построенных моделей законов обычно используют критерии согласия. Как правило, критерии используются в условиях проверки сложных гипотез (проверка осуществляется по той же выборке, по которой оценивались параметры закона). В таких ситуациях распределения статистик критериев чаще всего неизвестны, что является частым источником некорректности выводов. Третья проблема связана с качеством статистических данных. Хорошую модель часто удается построить и по результатам измерений, фиксируемым с существенными ошибками округления, а использование статистического критерия в той же ситуации может привести к неверному выводу, отклоняющему эту модель.

На основании качественных статистических данных (отражающих закономерности) измерений скорости ветра и солнечной инсоляции можно строить более точные модели законов распределения вероятностей для каждого из возобновляемых источников энергии.

Используя эти модели и математические соотношения, связывающие скорость ветра с мощностью ветроэнергетической установки (ВЭУ) и солнечную инсоляцию с мощностью, выдаваемой солнечной панелью, можно, используя статистическое моделирование, строить модели законов распределения для мощностей, генерируемых ВЭУ и солнечной электростанцией, соответственно.

Таким же образом на базе уже этих моделей можно моделировать распределение вероятностей мощности, генерируемой гибридной системой заданной конфигурации, для чего нет принципиальных трудностей. Это, в свою очередь, открывает возможность использовать полученные закономерности при проектировании гибридной системы, например, для оптимального подбора параметров накопителя, как резервирующего источника. А при существующей гибридной системе появляется возможность для прогнозирования и учета этой информации системным оператором энергосистемы в процессе принятия решений.

\section{Построение моделей законов распределения для ско- рости ветра}

В настоящей работе использовались выборки с результатами измерений скорости ветра $V$ по месяцам, зафиксированные на высоте 10 м в районе Кордай, Казахстан, в январе-декабре 2016 г. Объёмы выборок от 696 до 744 измерений скорости ветра в м/с с округлением до 0,01. И даже при такой точности в выборках оказывается достаточно много повторяющихся значений.

Анализ рядов измерений осуществлялся в рамках програмы системы ISW [26].

Эмпирические распределения, соответствующие выборкам скоростей ветра по месяцам, существенно отличаются. Достаточно близкими оказались лишь эмпирические распределения скорости ветра, соответствующие июлю и августу (рис. 1). Не вдаваясь в подробности, лишь в этом случае гипотеза об однородности законов не отклонялась ни по одному из девяти применяемых критериев однородности законов [27].

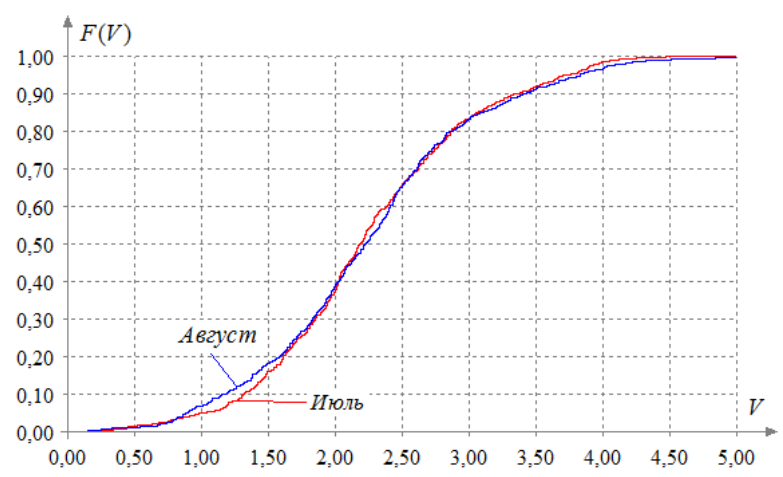

Pис. 1. Эмпирические функиии распределения скорости ветра за июль и август

Fig. 1. Empirical distribution functions of wind speed for July and August

Как правило, рассматриваемые выборки представляют собой временные ряды, не свободные от наличия тренда. В частности, для скорости ветра в июле гипотеза об отсутствии тренда в математическом ожидании отклоняется по большинству используемых критериев (есть тренд в математическом ожидании), а гипотеза об отсутствии тренда в дисперсии не отклоняется (нет тренда в дисперсии).

Тем не менее на практике, как правило, закрывают глаза на нарушение предпосылок, дающих основание для рассмотрения таких выборок, как выборки независимых одинаково распределенных случайных величин, считая, что такие нарушения не приведут к существенным ошибкам при проектировании или прогнозировании.

При построении моделей часто ограничиваются достаточно узким перечнем параметрических моделей законов распределений. В настоящем случае сгладить с достаточной точностью эмпирические распределения скоростей ветра, соответствующих рассматриваемым выборкам, параметрическими моделями законов распределения, используемыми различными авторами (Вейбулла [11, 12], бетараспределением I рода [13], Вейбулла, Максвелла или логарифмически нормальным [12]) оказалось невозможно. Более того, как правило, представленные выборки не удавалось сгладить ни одним законом, из включенных в программную систему ISW [26], в рамках которой предусмотрена возможность применения 
для описания реальных случайных величин более 30 различных параметрических моделей законов.

Исключение составили только распределения скорости ветра в сентябре и декабре, для которых достаточно хорошей для практического использования моделью оказались бета-распределения III рода с плотностью

$$
f(v, \theta)=\frac{\theta_{2}^{\theta_{0}}}{\theta_{3} \mathrm{~B}\left(\theta_{0}, \theta_{1}\right)} \frac{\left(\frac{v-\theta_{4}}{\theta_{3}}\right)^{\theta_{0}-1}\left(1-\frac{v-\theta_{4}}{\theta_{3}}\right)^{\theta_{1}-1}}{\left[1+\left(\theta_{2}-1\right) \frac{v-\theta_{4}}{\theta_{3}}\right]^{\theta_{0}+\theta_{1}}} .
$$

В остальных случаях хорошие модели удалось построить в виде смеси двух параметрических законов с плотностью

$$
f_{v}(v)=\alpha f_{1}\left(v, \theta_{1}\right)+(1-\alpha) f_{2}\left(v, \theta_{2}\right),
$$

где $f_{1}\left(v, \theta_{1}\right)$ и $f_{2}\left(v, \theta_{2}\right)$ - два бета-распределения III рода вида (1) со своими векторами параметров, а $\alpha$ - параметр смеси. Информация об оценках параметров моделей, построенных по выборкам скоростей ветра методом максимального правдоподобия, представлена в первой части табл. 1.

При наличии статистических данных за несколько лет можно было бы рассмотреть законы распределения в те же месяцы по годам и сравнить их с законом по объединенной выборке для соответствующего месяца, что позволило бы сделать выводы об устойчивости построенных моделей и перспективах их ис- пользования. Но в нашем случае таких статистических данных не было.

\section{Проверка адекватности построенных моделей}

Насколько хорошо полученные модели законов распределения соответствуют эмпирическим распределениям, построенным по соответствующим выборкам, как правило, проверяют с использованием критериев согласия. Для этого применяют непараметрические критерии согласия (Колмогорова, КрамераМизеса-Смирнова, Адерсона-Дарлинга, Купера, Ватсона, Жанга) [28] и критерии типа $\chi^{2}$.

В случае проверки простых гипотез вида: $H_{0}$ : $F(x)=F(x, \theta)$, где $F(x, \theta)$ - функция распределения вероятностей, с которой проверяют согласие наблюдаемой выборки, а $\theta$ - известное значение параметра (скалярного или векторного), распределения статистик непараметрических критериев согласия не зависят от вида $F(x, \theta)$.

При сложной проверяемой гипотезе вида: $H_{0}$ : $F(x) \in\{F(x, \theta), \theta \in \Theta)\}$ где $\Theta$ - область определения параметра $\theta$, а сам параметр оценивается по этой же выборке, «свобода от распределения» теряется [29] и на распределения статистик при справедливости $H_{0}$ влияет целый ряд факторов [28]: вид наблюдаемого закона распределения $F(x, \theta)$, соответствующего проверяемой гипотезе $H_{0}$; тип оцениваемого параметра и число оцениваемых параметров; в некоторых ситуациях конкретное значение параметра (например, в случае бета-распределений); используемый метод оценивания параметров.

Таблица 1. Параметры моделей законов распределения для скорости ветра и для мощности ветроэнергетической установки (кBm)

\begin{tabular}{|c|c|c|c|c|c|c|c|c|c|c|c|}
\hline \multirow{2}{*}{ Месяц/Month } & \multirow{2}{*}{$\alpha$} & \multicolumn{5}{|c|}{ Параметры/Parameters $f_{1}(\cdot, \theta)$} & \multicolumn{5}{|c|}{ Параметры/Parameters $f_{2}(\cdot, \theta)$} \\
\hline & & $\theta_{0}$ & $\theta_{1}$ & $\theta_{2}$ & $\theta_{3}$ & $\theta_{4}$ & $\theta_{0}$ & $\theta_{1}$ & $\theta_{2}$ & $\theta_{3}$ & $\theta_{4}$ \\
\hline \multicolumn{12}{|c|}{ для скорости ветра/for wind speed } \\
\hline Январь/January & 0,7 & 4,6371 & 1,7207 & 4,2316 & 3,65 & 0,0 & 5,7815 & 4,0476 & 0,2732 & 3,65 & 0,0 \\
\hline Февраль/February & 0,7 & 2,8273 & 1,7900 & 1,8594 & 3,89 & 0,0 & 11,8997 & 11,9717 & 0,3565 & 3,89 & 0,0 \\
\hline Март/March & 0,7 & 3,5153 & 3,2546 & 1,9239 & 4,8 & 0,0 & 9,9448 & 12,2312 & 0,9346 & 4,8 & 0,0 \\
\hline Апрель/April & 0,8 & 3,0739 & 3,3694 & 1,2079 & 4,8 & 0,0 & 1,7631 & 9,2106 & 0,2084 & 4,25 & 0,0 \\
\hline Май/Мау & 0,7 & 5,8472 & 1,4677 & 9,1134 & 5,79 & 0,0 & 9,6257 & 12,3347 & 1,2369 & 5,2 & 0,0 \\
\hline Июнь/June & 0,75 & 3,6752 & 8,7057 & 0,8795 & 5,7 & 0,0 & 14,9720 & 16,0427 & 0,4352 & 3,4 & 0,0 \\
\hline Июль/July & 0,8 & 3,1975 & 8,7547 & 0,4867 & 5,6 & 0,0 & 13,8362 & 15,0917 & 0,5183 & 3,2 & 0,0 \\
\hline Август/August & 0,8 & 4,0660 & 3,1998 & 1,9021 & 5,4 & 0,0 & 17,9074 & 9,1522 & 0,9908 & 3,4 & 0,0 \\
\hline Сентябрь/September & 1,0 & 3,5093 & 7,5721 & 0,6030 & 4,3710 & 0,0 & - & - & - & - & - \\
\hline Октябрь/October & 0,6 & 11,5563 & 1,8370 & 14,5462 & 5,3 & 0,0 & 5,5213 & 2,5501 & 0,8728 & 3,25 & 0,0 \\
\hline Ноябрь/November & 0,85 & 2,9527 & 12,3730 & 0,3815 & 6,32 & 0,0 & 21,1167 & 9,0512 & 7,2762 & 4,0941 & 0,0 \\
\hline Декабрь/December & 1,0 & 1,4690 & 12,0011 & 0,0889 & 5,0461 & 0,0 & - & - & - & - & - \\
\hline \multicolumn{12}{|c|}{ для мощности ветроэнергетической установки (кВт)/for wind turbine power $(\mathrm{kW})$} \\
\hline Январь/January & 0,71 & 0,6477 & 2,0301 & 2,6545 & 62,0 & 0,0 & 5,3209 & 4,1363 & 1,0 & 62,0 & 0,0 \\
\hline Февраль/February & 0,9 & 0,6436 & 3,8184 & 0,6115 & 83,2063 & 0,0 & 19,9094 & 37,7045 & 1,0 & 83,0 & 0,0 \\
\hline Март/March & 0,919 & 0,9282 & 1,9370 & 5,7423 & 116,0 & 0,0 & 3,2117 & 3,8638 & 1,0 & 26,0 & 6,0 \\
\hline Апрель/April & 0,91 & 0,8118 & 2,7964 & 2,0837 & 90,0 & 0,0 & 3,4166 & 3,1851 & 1,0 & 90,0 & 0,0 \\
\hline Май/Мау & 0,95 & 1,0165 & 0,9124 & 24,5866 & 200,0 & 0,0 & 7,9998 & 41,9298 & 1,0 & 80,0 & 0,0 \\
\hline Июнь/June & 0,835 & 0,9491 & 1,9417 & 1,0 & 31,0 & 0,0 & 0,4583 & 1,8608 & 1,0 & 131,95 & 0,0 \\
\hline Июль/July & 1,0 & 1,2438 & 2,2193 & 5,2029 & 148,26 & 0,0 & - & - & - & - & - \\
\hline Август/August & 0,89 & 1,0847 & 1,6845 & 6,7612 & 160,0 & 0,0 & 2,7943 & 1,6665 & 1,0 & 25,0 & 0,0 \\
\hline Сентябрь/September & 1,0 & 1,0297 & 11,1550 & 0,0 & - & - & - & - & $\begin{array}{llll}- & & & \\
\end{array}$ & - & - \\
\hline Октябрь/October & 0,82 & 1,0168 & 1,5016 & 14,2854 & 206,376 & 0,0 & 4,2970 & 4,0674 & 1,0 & 38,0 & 0,0 \\
\hline Ноябрь/November & 0,98 & 0,6386 & 10,0786 & 1,12401 & 319,103 & 0,0 & 1,0 & 1,0 & 1,0 & 320,0 & 0,0 \\
\hline Декабрь/December & 0,91 & 0,6211 & 2,0276 & 1,2010 & 129,5 & 0,0 & 7,0 & 47,0 & $\begin{array}{ll}- \\
\end{array}$ & - & - \\
\hline
\end{tabular}

Table 1. Parameters of models of distribution laws for wind speed and for wind turbine power $(k W)$ 
В нашем случае должна проверяться сложная гипотеза, причём в самой неблагоприятной ситуации. Проблема усугубляется тем, что неизвестные распределения статистик непараметрических критериев согласия зависят от конкретных значений трех параметров формы каждого из бета-распределений, входящих в смесь, и от значения параметра смеси, то есть от оценок семи параметров. Найти в такой ситуации методами статистического моделирования требуемые распределения статистик критериев, как это обычно делается в более простых случаях, оказывается очень непростой задачей, связанной с рядом вычислительных трудностей.

Пренебрегая фактом оценивания параметров по выборке, будем считать, что проверяется простая гипотеза. Тогда при проверке согласия по критериям Колмогорова, Крамера-Мизеса-Смирнова, Адерсона-Дарлинга, Купера и Ватсона, например, для января, выборки скорости ветра объёмом $n=744$ с построенной моделью

$$
f_{v}(x)=0,7 f_{1}\left(v, \theta_{1}\right)+0,3 f_{2}\left(v, \theta_{2}\right),
$$

где параметры для $f_{1}\left(v, \theta_{1}\right)$ и $f_{2}\left(v, \theta_{2}\right)$ берутся из первой строки табл. 1, получим результаты, представленные в табл. 2.

Таблица 2. Результаты проверки простой гипотезы о скорости ветра в январе

Table 2. Results of testing a simple hypothesis about wind speed in January

\begin{tabular}{|l|c|c|}
\hline \multicolumn{1}{|c|}{ Критерий/Test } & Статистика/Statistics & $p_{\text {value }}$ \\
\hline $\begin{array}{l}\text { Колмогорова } \\
\text { Kolmogorov }\end{array}$ & 0,51958 & 0,950 \\
\hline $\begin{array}{l}\text { Крамера-Мизеса-Смирнова } \\
\text { Cramer-Mises-Smirnov }\end{array}$ & 0,02533 & 0,989 \\
\hline $\begin{array}{l}\text { Aдерсона-Дарлинга } \\
\text { Aderson-Darling }\end{array}$ & 0,24157 & 0,975 \\
\hline $\begin{array}{l}\text { Kупера } \\
\text { Kuiper }\end{array}$ & 0,85775 & 0,952 \\
\hline $\begin{array}{l}\text { Baтcoна } \\
\text { Watson }\end{array}$ & 0,02517 & 0,965 \\
\hline
\end{tabular}

Приводимые значения достигнутого уровня значимости $p_{\text {value }}$ по всем критериям свидетельствуют о близости эмпирического распределения и функции распределения построенной модели (рис. 2). В то же время, так как на самом деле проверяется сложная гипотеза, приведенные значения $p_{\text {value }}$ являются завышенными.

Убедимся в качестве построенных моделей ещё одним несколько необычным способом. В соответствии с построенной моделью для закона распределения скорости ветра в январе смоделируем выборку такого же объема $n=744$ с округлением значений до 0,01 (как и в анализируемых выборках скорости ветpa).

Далее проверим гипотезу об однородности законов распределений смоделированной выборки и исходной выборки измерений скорости ветра в январе с использованием совокупности критериев проверки гипотез об однородности пары выборок [27, 30, 31], реализованных в системе [26] (критериями ЛеманаРозенблатта [32, 33], Андерсона-Дарлинга-Петита [34], модифицированным Смирнова [35, 36], Жанга (со статистиками Za, Zc, Zk) $[37,38])$. Результаты такой проверки, сделанные в [26], представлены в табл. 3. Как можно судить по достигнутым уровням значимости, оснований для отклонения гипотезы об однородности нет.

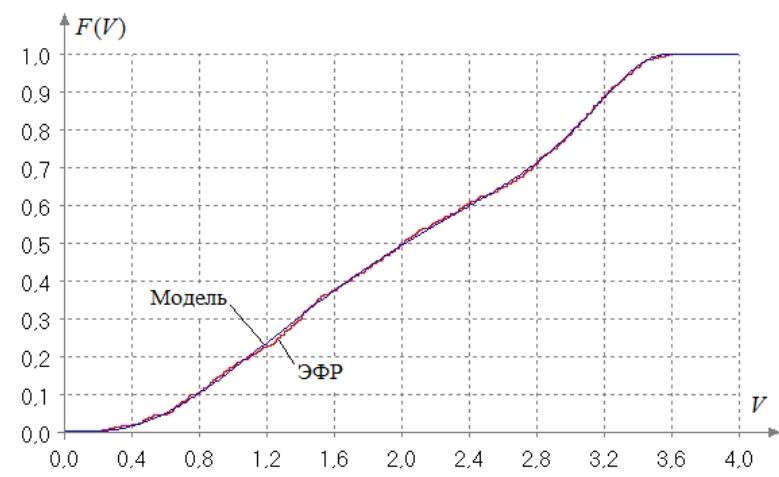

Pис. 2. Эмпирическая функиия распределения и модель функиии распределения скорости ветра в январе

Fig. 2. Empirical distribution function of wind speed and its distribution function model in January

Таблица 3. Результаты проверки гипотезы об однородности законов исходной и смоделированной выборок скорости ветра в январе

Table 3. Results of testing the hypothesis of homogeneity of laws original and simulated wind speed samples in January

\begin{tabular}{|l|c|c|}
\hline \multicolumn{1}{|c|}{ Критерий/Test } & $\begin{array}{c}\text { Статистика } \\
\text { Statistics }\end{array}$ & $p_{\text {value }}$ \\
\hline $\begin{array}{l}\text { Лемана-Розенблатта } \\
\text { Lehmann-Rosenblatt }\end{array}$ & 0,6438 & 0,6076 \\
\hline $\begin{array}{l}\text { Андерсона-Дарлинга-Петита } \\
\text { Aderson-Darling-Pettitt }\end{array}$ & 0,1020 & 0,5752 \\
\hline $\begin{array}{l}\text { Смирнова модиф. } \\
\text { Smirnov modif. }\end{array}$ & 0,7371 & 0,6488 \\
\hline Za Жанга / Za Zhang & 3,2813 & 0,1704 \\
\hline Zc Жанга / Zc Zhang & 3,2704 & 0,1917 \\
\hline Zk Жанга / Zk Zhang & 4,5197 & 0,1104 \\
\hline
\end{tabular}

\section{Построение моделей распределений мощности} ветроэнергетической установки

Одновременно с данными измерений скорости ветра в нашем распоряжении оказались выборки с «замерами мощности ВЭУ» $P$ также за январьдекабрь по месяцам 2016 г. Действуя аналогичным образом, по этим выборкам были построены параметрические модели законов распределения, информация о которых сконцентрирована во второй части табл. 1. И в данном случае наиболее предпочтительными моделями законов распределения для этих выборок также оказались смеси бета-распределений III рода за следующими исключениями:

- в июле закон распределения мощности ВЭУ достаточно хорошо описывается бета-распределением III рода (без смеси);

- в сентябре - усеченным обобщенным нормальным законом (или обобщенным полунормальным законом) с плотностью 


$$
f(p, \theta)=\frac{\theta_{0}}{\theta_{1} \Gamma\left(1 / \theta_{0}\right)} \exp \left\{-\left(\frac{\left|p-\theta_{2}\right|}{\theta_{1}}\right)^{\theta_{0}}\right\} ;
$$

- в декабре - смесью бета-распределения III рода вида (1) и нормального закона с масштабным параметром $\theta_{0}=7$ и сдвига $\theta_{1}=47$.

При проверке адекватности моделей, построенных для законов функций распределения мощности, возникают те же самые проблемы, что и при проверке моделей для скоростей ветра, и разрешаются они таким же образом: можно посмотреть на результаты проверки простых гипотез и убедиться, что моделирование выборок в соответствии с построенными моделями дают выборки, однородные исходным.

\section{О связи законов распределения скорости ветра и} мощности ветроэнергетической установки

Возможность построения закона распределения мощности ВЭУ на основании знания законов распределения скорости ветра, имеющих место в конкретной местности, вызывает серьёзный практический интерес.

Известно, что зная закон распределения (плотность) системы случайных величин $f_{x}\left(x_{1}, \ldots, x_{m}\right)$, можно найти закон распределения (плотность) $f_{y}(y)$ для функции $y=\psi\left(x_{1}, \ldots, x_{m}\right)$. Однако решение такой задачи в аналитическом виде находится в крайне редких ситуациях, лишь для некоторых $f(\cdot)$ и некоторых видов функций $\psi(\cdot)$. В то же время, используя методы статистического моделирования и отталкиваясь от модели $f_{x}\left(x_{1}, \ldots, x_{m}\right)$, на основании результатов моделирования можно строить модель для $f_{y}(y)$.

Мощность ВЭУ в ваттах в зависимости от скорости ветра (в метрах) приближенно определяется по следующей формуле [39]:

$$
P=C_{p} \pi R^{2} 0,5 V^{3} \rho \eta_{\text {red }} \eta_{\text {gen }},
$$

где $C_{p}$ - коэффициент использования энергии ветра, безразмерная величина (в номинальном режиме для быстроходных ветряков его значение достигает величины $0,4-0,5) ; R$ - радиус ротора (м); $V$ - скорость воздушного потока $(\mathrm{м} / \mathrm{c}) ; \rho$ - плотность воздуха (кг/м3); $\eta_{\text {red }}$ - КПД редуктора; $\eta_{g e n}$ - КПД генератора.

В формуле мощности все величины, кроме радиуса ротора, носят приближенный характер.

В нашем распоряжении имеются выборки измерений мощности и скорости ветра. Но скорость ветра замерена на высоте 10 м, что значительно ниже ступицы ротора. В данном случае мы не знаем, каким образом (насколько точно в условиях конкретной местности) пересчитывается скорость ветра на высоту ступицы ротора (по существу не знаем диапазон изменения скорости ветра на высоте ступицы).

Нам оказались неизвестными также и все остальные величины, входящие в правую часть формулы (2) И в то же время хотелось бы знать, насколько точно формула (2) позволяет установить связь между законами распределения вероятностей скорости ветра и мощности ВЭУ. И можно ли на основании (2), зная закон распределения мощности, восстановить закон распределения скорости ветра.
Естественно, что основная задача заключается в том, чтобы по закону распределения скорости ветра найти распределение мощности ВЭУ.

Из соотношения (2) для мощности можно выразить скорость ветра:

$$
V=\sqrt[3]{P /\left(0,5 C_{p} \pi R^{2} \rho \eta_{\text {red }} \eta_{\text {gen }}\right)} .
$$

Опираясь на построенные модели распределения мощности в соответствующем месяце, можно смоделировать распределение скорости ветра в этом же месяце, подобрав $k=0,5 C_{p} \pi R^{2} \rho \eta_{\text {red }} \eta_{g e n}$ таким образом, чтобы область определения случайной величины $V$ совпала с областью определения в соответствующей выборке для скорости ветра.

В качестве примера рассмотрим мощность ВЭУ и скорость ветра в январе. Закон распределения мощности в январе описывается смесью

$$
f_{p}(p)=0,71 f_{1}\left(p, \theta_{1}\right)+0,29 f_{2}\left(p, \theta_{2}\right),
$$

где $f_{1}\left(p, \theta_{1}\right)$ и $f_{2}\left(p, \theta_{2}\right)$ - бета-распределения III рода, параметры которых представлены в первой строке второй части табл. 1 . В $f_{1}\left(p, \theta_{1}\right)$ параметры $\theta_{1}=2,0301$, $\theta_{2}=2,6545, \theta_{3}=62,0, \theta_{4}=0,0$, в $f_{2}\left(p, \theta_{2}\right)-\theta_{0}=5,3209$, $\theta_{1}=4,1363, \theta_{2}=1,00, \theta_{3}=62,0, \theta_{4}=0,0$.

Далее, моделируя скорость ветра $V=\sqrt[3]{P / k}$ как функцию от случайной величины $P$ и подбирая величину $k$, будем получать различные эмпирические распределения для скорости ветра. Возможность моделирования законов распределения функций от случайных величин (моделирования выборок значений функции от случайных величин), принадлежащих различным законам, реализована в системе [26] и использована в данном случае. При $k=1,19$ моделируемое эмпирическое распределение функции $V=\sqrt[3]{P / k}$ практически совпадает с выборкой для скорости ветра в январе, как видно из рис. 2,3 .

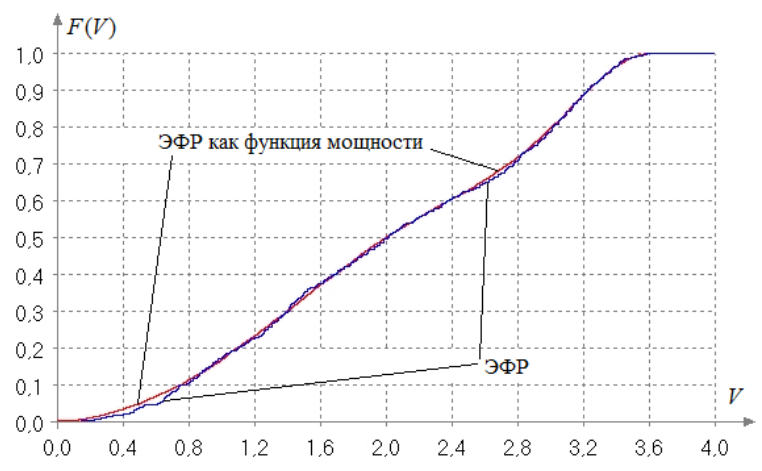

Pис. 3. Эмпирическое распределение скорости ветра в январе и эмпирическое распределение скорости ветра, полученное при моделировании функиии от мошности

Fig. 3. Empirical distribution of wind speed in January and the wind speed empirical distribution as a function of power

Этот факт свидетельствует о качестве построенных моделей, а значение $k=1,19$ в некоторой степени характеризует неизвестные нам параметры ВЭУ и 
изменение скорости ветра по высоте. По существу, отталкиваясь от построенной модели закона распределения мощности, мы восстановили закон распределения скорости ветра.

Таким же образом и так же хорошо распределения величины $V=\sqrt[3]{P / 1,19}$, как функции от случайной величины мощности $P$, согласуются с соответствующими выборками скорости ветра $V$ в другие месяцы года.

Зная закон распределения скорости ветра $V$, можно получить распределение мощности, моделируя её как функцию $P=1,19 V^{3}$ от случайной величины $V$. Например, в январе (табл. 1) скорость ветра описывается смесью двух бета-распределений III рода с плотностью

$$
f_{v}(v)=0,7 f_{1}\left(v, \theta_{1}\right)+0,3 f_{2}\left(v, \theta_{2}\right),
$$

где в $f_{1}\left(v, \theta_{1}\right)$ параметры принимают значения $\theta_{0}=4,6371, \theta_{1}=1,7207, \theta_{2}=4,2316, \theta_{3}=3,65, \theta_{4}=0,0$, а в $f_{2}\left(v, \theta_{2}\right) \quad-\theta_{0}=5,7815, \quad \theta_{1}=4,0476, \quad \theta_{2}=0,2732, \quad \theta_{3}=3,65$, $\theta_{4}=0,0$.

В данном случае смоделированное эмпирическое распределение мощности как функции скорости ветра практически совпадает с эмпирическим распределением мощности в январе (рис. 4).

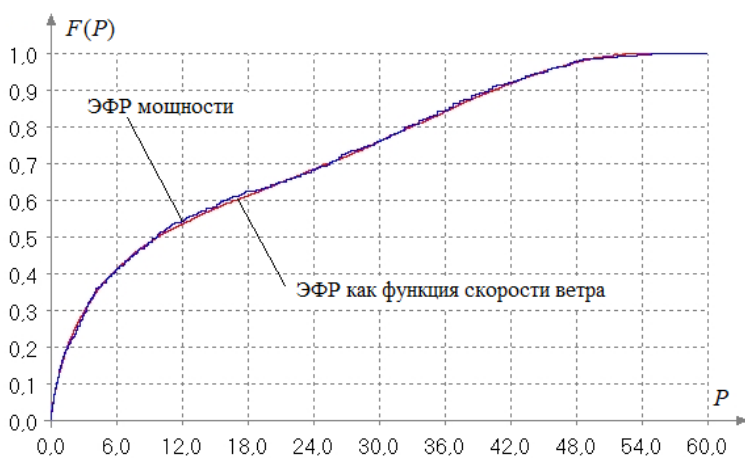

Рис. 4. Эмпирическое распределение мощчности в январе и эмпирическое распределение мощуности как функиии скорости ветра

Fig. 4. Empirical power distribution in January and empirical power distribution as a function of wind speed

Данный вычислительный эксперимент косвенно подтвердил качество построенных моделей для законов распределения ветра и мощности. В то же время его результаты вызвали сомнение, что имеющиеся выборки мощности представляют собой результаты измерений. Источник подтвердил, что выборки с данными по мощности получены на основании измерений скорости ветра по соотношению (2) без учета того, что при малых скоростях ветра ВЭУ могла выдавать нулевую мощность, а при штормовых - отключаться в целях безопасности. Учёт этих факторов при моделировании и более точное знание параметров в формуле (2) позволяет на основании законов распределения ветра строить законы распределения мощности для различных типов ВЭУ.
Если известны все характеристики ВЭУ и плотность воздуха $\rho$ (то есть $k$ известно), то на основании распределения мощности можно получить распределение скорости ветра на высоте ступицы ротора.

Если для ВЭУ известны КПД редуктора $\eta_{\text {red }}$ и генератора $\eta_{g e n}$, а также плотность воздуха $\rho$, то на основании выражения для $k$ можно оценить реальный коэффициент использования энергии ветра $C_{p}$ для данного типа ВЭУ.

В целом же проведенные вычислительные эксперименты демонстрируют реальную возможность того, как с использованием технологии статистического моделирования на основании модели распределения скорости ветра $f_{v}(v)$ для системы из $m$ ВЭУ с различными техническими характеристиками, используемыми в соотношении (2), можно построить хорошую модель для распределения вероятностей $f_{p_{\Sigma}}\left(p_{\Sigma}\right)$ мощности этой системы $P_{\Sigma}=\sum_{i=1}^{m} P_{i}(V)$.

\section{Модели законов распределения для инсоляции и} мощности солнечных элементов

Законы распределения инсоляции и мощности солнечных панелей (СП) и построенные для них модели оказываются очень похожими. Можно считать, что они отличаются практически только параметрами масштаба. Модели законов распределения для инсоляции и мощности СП, в основном в виде смесей бета-распределений III рода, построенные по результатам измерений в том же территориальном районе, представлены в первой и второй частях табл. 4, соответственно.

В частности, моделями в виде бета-распределений III рода (без смеси) описываются распределения инсоляции в апреле, мае, июне, июле и августе, а распределения мощности солнечной панели - в мае, июле и августе.

Подчеркнём, что, зная закон распределения вероятностей (модель) для солнечной инсоляции (в конкретной географической точке) и используя математические модели, связывающие величину инсоляции с мощностью солнечной панели или станции, можно моделировать законы распределения вероятностей для мощности солнечных батарей и солнечных станций, а также строить (хорошие) модели для этих законов.

Более того, реализованные в системе [26] возможности позволяют исследовать распределения функций от системы независимых случайных величин. Следовательно, в предположении о независимости случайных величин скорости ветра и солнечной инсоляции, опираясь на законы распределения скорости ветра и солнечной радиации, можно моделировать распределение вероятностей для мощности гибридной системы, состоящей из ряда ВЭУ и солнечных батарей, и строить модели для этого распределения. 
Таблица 5. Параметры моделей законов распределения для инсоляции (Bm/ $\left.\mathrm{m}^{2}\right)$ и для мощности солнечной панели (кBm)

Table 5. Parameters of models of distribution laws for insolation $\left(\mathrm{W} / \mathrm{m}^{2}\right)$ and for solar panel power $(\mathrm{kW})$

\begin{tabular}{|c|c|c|c|c|c|c|c|c|c|c|c|}
\hline \multirow{2}{*}{ Месяц/Month } & \multirow{2}{*}{$\alpha$} & \multicolumn{5}{|c|}{ Параметры/Parameters $f_{1}(\cdot ; \theta)$} & \multicolumn{5}{|c|}{ Параметры/Parameters $f_{2}(\cdot, \theta)$} \\
\hline & & $\theta_{0}$ & $\theta_{1}$ & $\theta_{2}$ & $\theta_{3}$ & $\theta_{4}$ & $\theta_{0}$ & $\theta_{1}$ & $\theta_{2}$ & $\theta_{3}$ & $\theta_{4}$ \\
\hline \multicolumn{12}{|c|}{ для инсоляции $\left(\mathrm{BT} / \mathrm{m}^{2}\right) /$ for insolation $\left(\mathrm{W} / \mathrm{m}^{2}\right)$} \\
\hline Январь/January & 0,8 & 2,8317 & 1,1313 & 21,3069 & 840,0 & 0,0 & 1,0206 & 1,5209 & 0,1707 & 840,0 & 0,0 \\
\hline Февраль/February & 0,75 & 2,2348 & 0,8947 & 11,6526 & 960,0 & 0,0 & 7,1219 & 0,6824 & 3,6042 & 960,0 & 0,0 \\
\hline Март/March & 0,71 & 1,2785 & 0,6734 & 7,4981 & 985,0 & 0,0 & 5,4617 & 1,7442 & 1,00 & 1050,0 & 0,0 \\
\hline Апрель/ April & 1,0 & 0,8045 & 0,6380 & 2,1939 & 1050,0 & 0,0 & - & - & - & - & - \\
\hline Май/Мау & 1,0 & 0,5302 & 0,8263 & 0,9361 & 1050,0 & 0,0 & - & - & - & - & $\begin{array}{llll}- & \end{array}$ \\
\hline Июнь/June & 1,0 & 0,6157 & 0,6164 & 1,4798 & 1015,0 & 0,0 & - & - & - & - & - \\
\hline Июль/July & 1,0 & 0,6804 & 0,5671 & 2,00 & 1000,0 & 0,0 & - & $\begin{array}{c}- \\
-\end{array}$ & - & - & - \\
\hline Август/August & 1,0 & 0,5538 & 0,7140 & 0,50 & 1036,0 & 0,0 & - & - & - & - & - \\
\hline Сентябрь/September & 0,6 & 0,7758 & 2,6445 & 0,0710 & 1050,0 & 0,0 & 1,2185 & 2,0540 & 1,6779 & 1050,0 & 0,0 \\
\hline Октябрь/October & 0,7 & 1,4650 & 0,7492 & 12,0948 & 985,0 & 0,0 & 2,2150 & 1,7268 & 0,4695 & 985,0 & 0,0 \\
\hline Ноябрь/November & 0,7 & 10,9080 & 2,2153 & 71,9885 & 882,0 & 0,0 & 24,5369 & 0,9226 & 19,4393 & 882,0 & 0,0 \\
\hline Декабрь/December & 0,7 & 1,0157 & 0,6871 & 5,8196 & 778,0 & 0,0 & 8,9954 & 2,2517 & 34,374 & 778,0 & 0,0 \\
\hline \multicolumn{12}{|c|}{ для мощности солнечной панели (кВт)/for solar panel power (kW) } \\
\hline Январь/January & 0,8 & 2,0474 & 0,8278 & 21,3674 & 0,6839 & 0,0 & 0,4133 & 3,8875 & 0,01736 & 0,6839 & 0,0 \\
\hline Февраль/February & 0,75 & 1,3448 & 0,9935 & 5,2486 & 0,764 & 0,0 & 8,0274 & 1,2118 & 1,5894 & $0,0,764$ & 0,0 \\
\hline Март/March & 0,81 & 0,7241 & 1,0253 & 1,8802 & 0,82 & 0,0 & 6,2835 & 5,7084 & 0,2659 & 0,836 & 0,0 \\
\hline Апрель/April & 0,8 & 0,6433 & 1,6090 & 0,7194 & 0,8374 & 0,0 & 5,8110 & 5,1488 & 0,2262 & 0,8649 & 0,0 \\
\hline Май/Мау & 1,0 & 0,4584 & 1,4019 & 0,3024 & 0,8161 & 0,0 & - & - & - & - & - \\
\hline Июнь/June & 0,7 & 0,6288 & 1,3854 & 1,2226 & 0,780 & 0,0 & 7,7521 & 1,1981 & 1,5408 & 0,756 & 0,0 \\
\hline Июль/July & 1,0 & 0,5362 & 0,6606 & 0,9384 & 0,741 & 0,0 & - & - & - & - & $\begin{array}{cc}- & \\
\end{array}$ \\
\hline Август/August & 1,0 & 0,4361 & 1,1241 & 0,1542 & 0,773 & 0,0 & $\begin{array}{llll}- & \end{array}$ & $\begin{array}{lllll}- & \end{array}$ & $\begin{array}{c}- \\
\end{array}$ & - & $\begin{array}{c}- \\
-\end{array}$ \\
\hline Сентябрь/September & 0,6 & 0,9720 & 2,6890 & 0,08296 & 0,776 & 0,0 & 0,9386 & 1,9253 & 1,5922 & 0,776 & 0,0 \\
\hline Октябрь/October & 0,7 & 1,1585 & 0,6229 & 11,8079 & 0,7715 & 0,0 & 2,0068 & 2,2514 & 0,2829 & 0,7715 & 0,0 \\
\hline Ноябрь/November & 0,7 & 1,8970 & 3,1671 & 8,9725 & 0,743 & 0,0 & 3,9328 & 1,4074 & 1,6728 & 0,743 & 0,0 \\
\hline Декабрь/December & 0,7 & 0,8088 & 0,6106 & 5,5542 & 0,645 & 0,0 & 5,4923 & 1,4970 & 34,8212 & 0,645 & 0,0 \\
\hline
\end{tabular}

\section{Определение числовых характеристик для распределений мощности ветроэнергетической установки}

Закон распределения случайной величины содержит полную информацию об этой случайной величине, включая числовые характеристики. Математическое ожидание для мощности энергетической установки определяет среднее значение генерируемой мощности, а дисперсия характеризует её рассеяние. В принципе, это те величины, на которые ориентируются на практике. В частности, на основании математического ожидания мощности вычисляются интегральные показатели для оценки выработки электроэнергии за длительный интервал времени.

Нахождение числовых характеристик для построенных достаточно сложных моделей законов распределения мощности (для ветровых и солнечных установок) в аналитическом виде может представлять собой непростую задачу, так как соответствующие интегралы для математического ожидания и дисперсии находятся только численными методами.

В рамках программной системы [26], имея модель закона распределения мощности $P$, несложно найти оценки математического ожидания, дисперсии и среднеквадратичного отклонения методами статистического моделирования. Для этого достаточно смоделировать выборку значений мощности $P_{1}, P_{2} \ldots, P_{n}$ в соответствии с моделью этого закона при желаемом объёме выборки $n$ и по этой выборке найти соответствующие оценки $\hat{E}[P]=\bar{P}=\frac{1}{n} \sum_{i=1}^{n} P_{i}, \hat{D}[P]=\frac{1}{n-1} \sum_{i=1}^{n}\left(P_{i}-\bar{P}\right)^{2}$ и $\hat{\sigma}_{P}=\sqrt{\hat{D}[P]}$.
Следует отметить, что законы распределения мощности ВЭУ в разные месяцы существенно отличаются. Иногда вид закона очень наглядно объясняет трудности прогнозирования и особенности погодных условий.

\section{Заключение}

Исследование законов распределения скоростей ветра и солнечной инсоляции опиралось на реальные и достаточно точные измерения этих величин в указанном выше районе в течение года.

Результаты показали, что выборки измерений скорости ветра и солнечной инсоляции не удаётся удовлетворительно описать законами распределения, наиболее часто упоминаемыми в различных источниках, как применяемыми в этих целях. Однако они хорошо описываются семейством бета-распределений 3-го рода и их смесями. Такие модели для законов распределения скорости ветра и солнечной инсоляции построены для всех 12 месяцев.

Показано, что на базе построенных моделей законов распределения для скорости ветра и солнечной инсоляции и соотношений, связывающих скорость ветра с мощностью ветроэнергетической установки, а инсоляцию с мощностью, выдаваемой солнечной панелью, можно моделировать распределения для мощности соответствующих устройств и строить модели законов распределения для них.

При моделировании распределения вероятностей для мощности конкретного типа ветроэнергетической установки должен быть задан интервал скоростей ветра, в котором обеспечивается возможность генерации этой установки. 
Реализованные в [26] возможности по моделированию законов распределения функций от случайных величин [40] позволяют на основании моделей законов распределения скорости ветра и солнечной инсоляции (на конкретной территории) моделировать законы распределения для мощности гибридных электроэнергетических систем различной конфигурации. Получаемые результаты могут использоваться при выборе и проектировании ветровых и солнечных

\section{СПИСОК ЛИТЕРАТУРЫ}

1. Elhadidy M.A., Shaahid S.M., Parametric study of hybrid (wind+solar+diesel) power generating systems // Renew Energy. 2000. - V. 21. - № 2. - P. 129-139.

2. Karaki S.H., Chedid R.B., Ramadan R. Probabilistic performance assessment of autonomous solar-wind energy conversion systems // IEEE Trans Energy Conversion. - 1999. - V. 14. - № 3 . - P. 766-772.

3. Borrowsy B.S., Salameh Z.M. Optimum photovoltaic array size for a hybrid wind/PV systems // IEEE Trans Energy Conversion. 1994. - V. 9. - № 3. - P. 482-488.

4. Kruangpradit P., Tayati W. Hybrid renewable energy system development in Thailand // Renewable Energy. - 1996. - V. 8. № 1-4. - P. 514-517.

5. Generation unit sizing and cost analysis for standalone wind photovoltaic and hybrid wind/PV systems / W.D. Kellogg, M.H. Nehrir, G. Venkataramana, V. Gerez // IEEE Trans Energy Conversion. - 1998. - V. 13. - № 1. - P. 70-75.

6. Chedid R., Rahman S. Unit sizing and control of hybrid wind-solar power system // IEEE Trans Energy Conversion. - 1997. V. 112. - № 1. - P. 79-86.

7. Optimization of hybrid solar wind power systems / E. Dilettoso, S. Gagliano, N. Salerno, G. Tina // International Journal of Applied Electromagnetics and Mechanics. - 2007. - V. 26. - № 3-4. P. 225-231.

8. Tina G., Gagliano S., Raiti S. Hybrid solar/wind power system probabilistic modelling for long-term performance assessment // Solar Energy. - 2006. - V. 80. - № 5. - P. 578-588.

9. Hollands K.T.G., Huget R.G. A probability density function for the clearness index, with applications // Solar Energy. - 1983. V. 30. - P. 195-209.

10. Ettoumi F.Y., Sauvageot H., Adane A.E.H. Statistical bivariate modelling of wind using first-order Markov chain and Weibull distribution // Renewable Energy. - 2003. - V. 28. - № 11. P. $1787-1802$.

11. Tina G., Gagliano S. Probability analysis of weather data for energy assessment of hybrid solar/wind power system // 4th IASME/WSEAS International Conference on Energy, Environment, Ecosystems and Sustainable Development (EEESD'08). - Algarve, Portugal, June 11-13, 2008. - P. 217-223.

12. Рыхлов А.Б. Анализ применения различных законов распределения для выравнивания скоростей ветра на юго-востоке европейской территории России // Известия Саратовского университета. - 2010. - Т. 10. - Сер. Науки о Земле. - № 2. C. $25-30$

13. Bashahu M., Buseke M. Statistical analysis of hourly wind speed data from some burundian stations using beta probability density functions // Modern Environmental Science and Engineering. 2016. - V. 2. - № 11. - P. 740-746.

14. Safari B. Modeling wind speed and wind power distributions in Rwanda // Renewable and Sustainable Energy Reviews. - 2011. V. 15. - № 2. - P. 925-935.

15. Mohammadi K., Alavi O., McGowan J.G. Use of BirnbaumSaunders distribution for estimating wind speed and wind power probability distributions: A review // Energy Conversion and Management. - 2017. - V. 143. - P. 109-122.

16. Wind speed analysis using the extended generalized Lindley distribution / Y.M. Kantar, I. Usta, I. Arik, I. Yenilmez // Renewable Energy. - 2018. - V. 118. - P. 1024-1030.

17. Aries N., Boudia S.M., Ounis H. Deep assessment of wind speed distribution models: a case study of four sites in Algeria // Energy Conversion and Management. - 2018. - V. 155. - P. 78-90. электростанций или гибридных электроэнергетических систем, состоящих из различных комбинаций ветровых и солнечных станций. Построенные по результатам такого моделирования модели законов распределения для генерируемых мощностей существующих гибридных систем могут использоваться при прогнозировании и учитываться [41] системным оператором энергосистемы в процессе принятия решений.

18. Jung C., Schindler D. Global comparison of the goodness-of-fit of wind speed distributions // Energy Conversion and Management. 2017. - V. 133. - P. 216-234.

19. Introducing a system of wind speed distributions for modeling properties of wind speed regimes around the world / C. Jung, D. Schindler, J. Laible, A. Buchholz // Energy Conversion and Management. - 2017. - V. 144. - P. 181-192.

20. Jung C., Schindler D. 3D statistical mapping of Germany's wind resource using WSWS // Energy Conversion and Management. 2018. - V. 159. - P. 96-108.

21. Ouarda T.B.M.J., Charron C., Chebana F. Review of criteria for the selection of probability distributions for wind speed data and introduction of the moment and L-moment ratio diagram methods, with a case study // Energy Conversion and Management. 2016. - V. 124 - P. 247-265.

22. Arthur Y.D., Gyamfi K.B., Appiah S.K. Probability distributional analysis of hourly solar irradiation in Kumasi-Ghana // International Journal of Business and Social Research. - 2013. V. 3. - № 3. - P. 63-75.

23. Guwaeder A., Ramakumar R. Statistical Analysis of PV Insolation Data // 2017 IEEE 44th Photovoltaic Specialist Conference (PVSC). - Washington, DC, 2017. - P. 1122-1126.

24. Solar PV grid power flow analysis / Q. Alsafasfeh, O. Saraereh, I. Khan, S. Kim // Sustainability. - 2019. - V. 11. - № 6. - 1744. DOI:10.3390/su11061744

25. Ayodele T.R. Determination of probability distribution function for modelling global solar radiation: case study of Ibadan, Nigeria // International Journal of Applied Science and Engineering. 2015. - V. 13. - № 3. - P. 233-245.

26. ISW - Программная система статистического анализа одномерных наблюдений. URL: https://ami.nstu.ru/ headrd/ISW.htm (дата обращения 03.02.2020).

27. Лемешко Б.Ю. Критерии проверки гипотез об однородности. Руководство по применению. - М.: ИНФРА-М, 2017. - 208 с. DOI: $10.12737 / 22368$

28. Лемешко Б.Ю. Непараметрические критерии согласия: Руководство по применению. - М.: ИНФРА-М, 2014. - 163 с. DOI: $10.12737 / 11873$

29. Kac M., Kiefer J., Wolfowitz J. On tests of normality and other tests of goodness of fit based on distance methods // The Annals of Mathematical Statistics. - 1955. - V. 26. - № 2. - P. 189-211.

30. Application of homogeneity tests: problems and solution / B.Y. Lemeshko, I.V. Veretelnikova, S.B. Lemeshko, A.Y. Novikova // Lecture Notes in Computer Science. - Cham: Springer, 2017. 10684. - P. 461-475. DOI: 10.1007/978-3-319-71504-9_38.

31. Лемешко Б.Ю., Веретельникова И.В. Мошность k-выборочных критериев проверки однородности законов // Измерительная техника. - 2018. - № 7. - C. 3-7. DOI: 10.32446/0368-1025it-2018-7-3-7

32. Lehmann E. L. Consistency and unbiasedness of certain nonparametric tests // The Annals of Mathematical Statistics. 1951. - V. 22. - № 1. - P. 165-179.

33. Rosenblatt M. Limit theorems associated with variants of the von Mises statistic // The Annals of Mathematical Statistics. - 1952. V. 23. - P. 617-623.

34. Pettitt A.N. A two-sample Anderson-Darling rank statistic // Biometrika. - 1976. - V. 63. - № 1. - P. 161-168.

35. Смирнов Н.В. Оценка расхождения между эмпирическими кривыми распределения в двух независимых выборках // Бюллетень МГУ, Серия А. - 1939. - Т. 2. - № 2. - С. 3-14

36. Лемешко Б.Ю., Лемешко С.Б. О сходимости распределений статистик и мощности критериев однородности Смирнова и 
Лемана-Розенблатта // Измерительная техника. - 2005. № 12. - C. $9-14$

37. Zhang J. Powerful two-sample tests based on the likelihood ratio // Technometrics. - 2006. - V. 48. - № 1. - P. 95-103. DOI $10.1198 / 004017005000000328$

38. Zhang J., Wu Y. k-Sample tests based on the likelihood ratio // Computational Statistics \& Data Analysis. - 2007. - V. 51. № 9. - P. 4682-4691.

39. Безруких П.П. Ветроэнергетика: справочное и методическое пособие. - М.: ИД «Энергия», 2010. - 195 с.

40. Blinov P.Y., Lemeshko B.Y. The simulation system and research of functions of random variables // 2016 13th International
Scientific-Technical Conference on Actual Problems of Electronics Instrument Engineering (APEIE). - Novosibirsk, 2016. - P. 203-209.

41. Повышение степени интеграции ветроэнергетических станций в энергосистему путем использования у системного оператора математических моделей прогнозирования ветра и электропотребления / В.З. Манусов, А.А. Холдонов, К.Н. Бойко, Ш.К. Халдаров // Проблемы региональной энергетики. - 2017. № 3 (35). - C. 45-57.

Поступила 02.09.2020 г.

\section{Информация об авторах}

Манусов В.3., доктор технических наук, профессор кафедры электроснабжения предприятий Новосибирского государственного технического университета.

Лемешко Б.Ю., доктор технических наук, профессор кафедры теоретической и прикладной информатики Новосибирского государственного технического университета.

Халдаров Ш.К., соискатель ученой степени кандидата наук, стажер кафедры электроснабжения предприятий Новосибирского государственного технического университета. 
UDC 621.311.25: 621.311.26

\section{MODELING OF DISTRIBUTION LAWS FOR WIND TURBINE AND SOLAR BATTERY OUTPUT POWER}

Vadim Z. Manusov'1,
Manusov36@mail.ru

Boris Yu. Lemeshko1, lemeshko@ami.nstu.ru

Sherzod K. Khaldarov ${ }^{1}$ haldaroff@mail.ru

1 Novosibirsk State Technical University, 20, Karl Marx avenue, Novosibirsk, 630073, Russia.

Relevance. The power provided by generating stations based on wind and solar energy depends on the state of the environment at a particular geographical point, the prevailing wind speeds and the level of solar insolation. The wind speeds and solar insolation characterizing patterns are probabilistic in nature and depend on the terrain and season. This means that the power provided by generating stations based on renewable energy sources is also probabilistic. Risks associated with an inaccurate knowledge of the probabilistic patterns describing renewable energy sources, as well as the unpredictability (uncertainty) of the natural environment affecting renewable energy sources, are the reasons why system operators do not take into account such stations in calculating the power balance. This situation does not allow considering the generating stations as full-fledged market participants, covering the flow of investments in such projects. Accurate determination of the output power distribution laws for solar and wind power stations, based on the wind speed and solar insolation data analysis can significantly help system operators in power predicting of the power stations.

Aim of study is building models of distribution laws that best describe the results of wind speed and solar insolation observations, as well as models of distribution laws for the generated power of wind and solar stations.

Methods: methods for estimating the parameters of distribution laws, methods for testing statistical hypotheses.

Results. It is shown, that the 3 rd kind of beta distribution laws and their mixtures describe the series of measured wind speed and solar insolation better in the territory. The same concerning the probability distribution of the wind and solar stations power. Distribution law models for wind speed, solar insolation and power of the corresponding stations are constructed. It is shown that on the basis of the law of the distribution of wind speed and the mathematical relationships that connect power with wind speed, good models can be constructed for the probability distributions of power of wind power stations. The models can be used as an auxiliary tool for power system operators to predict output power.

Key words:

Probability distribution laws, renewable energy sources, probabilistic output power, third kind of beta-distribution.

\section{REFERENCES}

1. Elhadidy M.A., Shaahid S.M. Parametric study of hybrid (wind+solar+diesel) power generating systems. Renew Energy, 2000, vol. 21, no. 2, pp. 129-139.

2. Karaki S.H., Chedid R.B., Ramadan R. Probabilistic performance assessment of autonomous solar-wind energy conversion systems. IEEE Trans Energy Conversion, 1999, vol. 14, no. 3, pp. 766-772.

3. Borrowsy B.S., Salameh Z.M. Optimum photovoltaic array size for a hybrid wind/PV systems. IEEE Trans Energy Conversion, 1994, vol. 9, no. 3, pp. 482-488.

4. Kruangpradit P., Tayati W. Hybrid renewable energy system development in Thailand. Renewable Energy, 1996, vol. 8, no. 1-4 pp. 514-517.

5. Kellogg W.D., Nehrir M.H., Venkataramana G., Gerez V. Generation unit sizing and cost analysis for standalone wind, photovoltaic and hybrid wind/PV systems. IEEE Trans Energy Conversion, 1998, vol. 13, no. 1, pp. 70-75.

6. Chedid R., Rahman S. Unit sizing and control of hybrid wind-solar power system. IEEE Trans Energy Conversion, 1997, vol. 112 no. 1, pp. 79-86.

7. Dilettoso E., Gagliano S., Salerno N., Tina G. Optimization of hybrid solar wind power systems. International Journal of Applied Electromagnetics and Mechanics, 2007, vol. 26, no. 3-4, pp. 225231.

8. Tina G., Gagliano S., Raiti S. Hybrid solar/wind power system probabilistic modelling for long-term performance assessment. Solar Energy, 2006, vol. 80, no. 5, pp. 578-588.

9. Hollands K.T.G., Huget R.G. A probability density function for the clearness index, with applications. Solar Energy, 1983, vol. 30, no. 3, pp. 195-209.
10. Ettoumi F.Y., Sauvageot H., Adane A.E.H. Statistical bivariate modelling of wind using first-order Markov chain and Weibull distribution. Renewable Energy, 2003, vol. 28, no. 11, pp. 17871802.

11. Tina G., Gagliano S. Probability analysis of weather data for energy assessment of hybrid solar/wind power system. $4^{\text {th }}$ IASME/WSEAS International Conference on Energy, Environment, Ecosystems and Sustainable Development (EEESD'08). Algarve, Portugal, June 11-13, 2008. pp. 217-223.

12. Rykhlov B.A. Analysis of the various distribution laws application to equalize wind speeds in the southeast of the Russian European territory. News of Saratov University, 2010, vol. 10, no. 2, Ser. Earth sciences, pp. 25-30. In Rus.

13. Bashahu M., Buseke M. Statistical analysis of hourly wind speed data from some burundian stations using beta probability density functions. Modern Environmental Science and Engineering, 2016, vol. 2, no. 11, pp. 740-746.

14. Safari B. Modeling wind speed and wind power distributions in Rwand. Renewable and Sustainable Energy Reviews, 2011, vol. 15, no. 2, pp. 925-935.

15. Mohammadi K., Alavi O., McGowan J.G. Use of BirnbaumSaunders distribution for estimating wind speed and wind power probability distributions: a review. Energy Conversion and Management, 2017, vol. 143, pp. 109-122

16. Kantar Y.M., Usta I., Arik I., Yenilmez I. Wind speed analysis using the extended generalized Lindley distribution. Renewable Energy, 2018, vol. 118, pp. 1024-1030.

17. Aries N., Boudia S.M., Ounis H. Deep assessment of wind speed distribution models: a case study of four sites in Algeria. Energy Conversion and Management, 2018, vol. 155, pp. 78-90. 
18. Jung C., Schindler D. Global comparison of the goodness-of-fit of wind speed distributions. Energy Conversion and Management, 2017, vol. 133, pp. 216-234.

19. Jung C., Schindler D., Laible J., Buchholz A. Introducing a system of wind speed distributions for modeling properties of wind speed regimes around the world. Energy Conversion and Management, 2017, vol. 144, pp. 181-192.

20. Jung C., Schindler D. 3D statistical mapping of Germany's wind resource using WSWS. Energy Conversion and Management, 2018, vol. 159, pp. 96-108.

21. Ouarda T.B.M.J., Charron C., Chebana F. Review of criteria for the selection of probability distributions for wind speed data and introduction of the moment and L-moment ratio diagram methods, with a case study. Energy Conversion and Management, 2016, vol. 124, pp. 247-265.

22. Arthur Y.D., Gyamfi K.B., Appiah S.K. Probability distributional analysis of hourly solar irradiation in Kumasi-Ghana. International Journal of Business and Social Research, 2013, vol. 3, no. 3, pp. 63-75.

23. Guwaeder A., Ramakumar R. Statistical analysis of PV insolation data. 2017 IEEE $44^{\text {th }}$ Photovoltaic Specialist Conference (PVSC). Washington, DC, 2017. pp. 1122-1126.

24. Alsafasfeh Q., Saraereh O., Khan I., Kim S. Solar PV grid power flow analysis. Sustainability, 2019, vol. 11, no. 6, 1744. DOI:10.3390/su11061744

25. Ayodele T.R. Determination of probability distribution function for modelling global solar radiation: case study of Ibadan, Nigeria. International Journal of Applied Science and Engineering, 2015, vol. 13 , no. 3, pp. 233-245.

26. ISW - Programmnava sistema statisticheskogo analiza odnomernykh nablyudeniy [ISW - Software system for statistica analysis of one-dimensional observations]. Available at: https://ami.nstu.ru/ headrd/ISW.htm (accessed 3 February 2020)

27. Lemeshko B.Yu. Kriterii proverki gipotez ob odnorodnosti. Rukovodstvo po primeneniyu [Tests for homogeneity. Guide on the application]. Moscow, INFRA-M Publ., 2017. 208 p. DOI: $10.12737 / 22368$

28. Lemeshko B.Yu. Neparametricheskie kriterii soglasiya: rukovodstvo po primeneniyu [Nonparametric goodness-of-fit tests. Guide on the application]. Moscow, INFRA-M Publ., 2014. 163 p. DOI: $10.12737 / 11873$

29. Kac M., Kiefer J., Wolfowitz J. On tests of normality and other tests of goodness of fit based on distance methods. The Annals of Mathematical Statistics, 1955, vol. 26, no. 2, pp. 189-211.
30. Lemeshko B.Y., Veretelnikova I.V., Lemeshko S.B., Novikova A.Y. Application of homogeneity tests: problems and solution. Lecture Notes in Computer Science. Cham, Springer, 2017. 10684, pp. 461-475. DOI: 10.1007/978-3-319-71504-9_38

31. Lemeshko B.Yu., Veretelnikova I.V. Power of k-sample tests aimed at checking the homogeneity of laws. Measurement Techniques, 2018, vol. 61, no. 7, pp. 647-654. DOI: 10.1007/s11018-018-1479-1

32. Lehmann E.L. Consistency and unbiasedness of certain nonparametric tests. The Annals of Mathematical Statistics, 1951, vol. 22, no. 1, pp. 165-179.

33. Rosenblatt M. Limit theorems associated with variants of the von Mises statistic. The Annals of Mathematical Statistics, 1952, vol. 23, no. 4, pp. 617-623.

34. Pettitt A.N. A two-sample Anderson-Darling rank statistic. Biometrika, 1976, vol. 63, no. 1, pp. 161-168.

35. Smirnov V.N. Otsenka raskhozhdeniya mezhdu impericheskimi krivymi raspredeleniya $\mathrm{v}$ dvukh nezavisimykh vyborkakh [Estimation of the discrepancy between empirical distribution curves in two independent samples]. Bulletin of Moscow State University, Series A, 1939, vol. 2, no. 2, pp. 3-14.

36. Lemeshko B.Yu., Lemeshko S.B. Statistical distribution convergence and homogeneity test power for Smirnov and Lehmann-Rosenblatt tests. Measurement Techniques, 2005, vol. 48, no. 12, pp. 1159-1166. In Rus.

37. Zhang J. Powerful two-sample tests based on the likelihood ratio. Technometrics, 2006, vol. 48, no. 1, pp. 95-103. DOI $10.1198 / 004017005000000328$

38. Zhang J., Wu Y. k-Sample tests based on the likelihood ratio. Computational Statistics \& Data Analysis, 2007, vol. 51, no. 9 , pp. 4682-4691.

39. Bezrukikh P.P. Vetroenergetika: spravochnoe $i$ metodicheskoe posobie [Wind energy: a reference and methodological manual]. Moscow, Energiya Publ., 2010. 195 p.

40. Blinov P.Y., Lemeshko B.Y. The simulation system and research of functions of random variables. $13^{\text {th }}$ International ScientificTechnical Conference on Actual Problems of Electronics Instrument Engineering (APEIE), Novosibirsk, 2016. pp. 203-209.

41. Manusov V.Z., Kholdonov A.A., Boyko K.N., Khaldarov S.K. Increasing the degree of integration of wind power stations in the power system by using mathematical models of wind forecasting and power consumption by the system operator. Problems of regional energy, 2017, vol. 3, no. 35, pp. 45-57. In Rus.

Received: 2 September 2020.

\section{Information about the authors}

Vadim Z. Manusov, Dr. Sc., professor, Novosibirsk State Technical University.

Boris Yu. Lemeshko, Dr. Sc., professor, Novosibirsk State Technical University.

Sherzod K. Khaldarov, applicant for Cand. Sc., trainee, Novosibirsk State Technical University. 